В.В. ЦУКАНОВ, Д.М.Н., профессор, Э.В. КАСПАРОВ, Д.М.Н., профессор, А.В. ВАСЮТИН, К.М.Н., Ю.Л. ТОНКИХ, К.М.Н., О.В. ПЕРЕТЯТЬКО Федеральный исследовательский центр «Красноярский научный центр» Сибирского отделения Российской академии наук, обособленное подразделение «Научно-исследовательский институт медицинских проблем Севера», Красноярск

\title{
ЭФФЕКТИВНОСТЬ РАБЕПРАЗОЛА
}

\section{ДЛЯ ЛЕЧЕНИЯ КИСЛОТОЗАВИСИМЫХ ЗАБОЛЕВАНИИ}

\begin{abstract}
Выполнен анализ современных данных об эффективности рабепразола в лечении кислотозависимых заболеваний. Европейский консенсус Маастрихт-5 рекомендует предпочтительное применение рабепразола в схемах эрадикации Helicobacter pylori. Клинические исследования подтвердили высокую эффективность рабепразола для лечения ГЭРБ и профилактики НПВП-гастропатий. Современным и эффективным дженериком рабепразола является препарат Рабиет ${ }^{\circledR}$

Ключевые слова: кислотозависимые заболевания, рабепразол, Helicobacter pylori, ГЭРБ.

V.V.TSUKANOV, MD, Prof., E.V. KASPAROV, MD, Prof., A.V.VASYUTIN, PhD in medicine, Y.L. TONKIKH, PhD in medicine, O.V. PERETYATKO Federal Research Center of Krasnoyarsk Scientific Center», Siberian branch of the Russian Academy of Sciences, separated subdivision «Scientific-research Institute of medical problems of the North», Krasnoyarsk

EFFECTIVENESS OF RABEPRAZOLE FOR THE TREATMENT OF ACID-RELATED DISEASES

The analysis of modern data about the effectiveness of rabeprazole in the treatment of acid-related diseases is performed. The European Consensus Maastricht 5 recommends a preferred use of rabeprazole in the schemes of eradication of Helicobacter pylori. Clinical studies confirmed the high efficiency of rabeprazole for the treatment of GERD, and prevention of NSAID-induced gastropathy. Modern and effective generic of rabeprazole is Rabiet.
\end{abstract}

Keywords: acid-related diseases, rabeprazole, Helicobacter pylori, GERD.

H аиболее актуальные аспекты применения ингибиторов протонной помпы (ИПП) для ведения пациентов с кислотозависимыми заболеваниями включают:

1. Эрадикацию Helicobacter pylori.

2. Терапию пациентов с гастроэзофагеальной рефлюксной болезнью (ГЭРБ).

3. Профилактику гастродуоденальных кровотечений у пациентов, систематически получающих нестероидные противовоспалительные препараты (НПВП) и антитромбоцитарные средства.

\section{РАБЕПРАЗОЛ И ЭРАДИКАЦИЯ HELICOBACTER PYLORI}

Эффективность эрадикации Helicobacter pylori зависит от многих факторов, среди которых имеет важное значение выбор ИПП [1]. В настоящее время доказано, что эффективность ингибиторов протонной помпы зависит от полиморфизма цитохрома СРР2С19. Большинство ингибиторов протонной помпы, включая омепразол, лансопразол и пантопразол, интенсивно метаболизируются в системе цитохрома CYP2C19 [2]. Однако эффективность эрадикации Helicobacter pylori с применением рабепразола достоверно выше, что доказано результатами различных метаанализов, в том числе современного метаанализа по 35 исследованиям (5 998 человек) и мультицентрового исследования в Японии, в ходе которого тройную семидневную терапию, включающую рабепразол, применяли для эрадикации Helicobacter pylori у 3162 человек и эффективность лечения составила 80,7\% [3-6]. В опубликованном в 2017 г. европейском консенсусе Маастрихт-5 официально рекомендуется применять преимущественно рабепразол и эзомепразол в схемах эрадикации первой и второй линии [7]. Таким образом, рабепразол можно считать надежным и эффективным ингибитором протонной помпы, действие которого не зависит от полиморфизма в системе CYP2C19.

Для практических врачей целесообразно повторить основные постулаты европейского консенсуса Маастрихт-5. В регионах с низкой резистентностью Helicobacter pylori $\mathrm{k}$ кларитромицину в качестве терапии первой линии может применяться тройная терапия (ИПП + кларитромицин + амоксицилин) в течение 14 дней. В регионах с резистентностью Helicobacter pylori к кларитромицину выше 15\% в качестве схемы первой линии для эрадикации рекомендуется квадротерапия с висмутом или конкомитантная терапия (ИПП + амоксицилин + кларитромицин + метронидазол). После неудачи терапии первой линии в качестве схемы второй линии целесообразны квадротерапия с висмутом (Новобисмол) или тройная терапия с фторхинолоном (ИПП + амоксицилин + левофлоксацин) или конкомитантная схема [7].

\section{РАБЕПРАЗОЛ И ЛЕЧЕНИЕ ГЭРБ}

В настоящее время ГЭРБ является самой частой патологией органов пищеварения [8, 9]. Наиболее эффективными препаратами для лечения патологии пищевода являются ИПП [10]. Вместе с тем в последние десятилетия возникла проблема резистентной к ИПП ГЭРБ. В настоящее время принято считать, что рефрактерной гастроэзофагеальной рефлюксной болезнью (ГЭРБ) является форма заболевания с персистированием типичных симптомов (изжога и/или регургитация), которые не отвечают на стабильный прием ингибитора протонной помпы 2 раза в сутки в течение не менее 12 недель [11]. К рефлюкс-ассоциированным причинам резистентной ГЭРБ относят персистирующий рефлюкс кислоты, вызванный неправильным назначением 
терапии или плохим комплаенсом, быстрый метаболизм ИПП у пациента, анатомические дефекты (грыжа пищеводного отверстия диафрагмы), персистирующий рефлюкс некислого содержимого из желудка или двенадцатиперстной кишки, гиперчувствительность пищевода к физиологическому количеству кислого, слабокислого или газового рефлюксата [12]. Выбор наиболее эффективного ИПП является принципиально важным моментом в алгоритме лечения рефрактерной ГЭРБ (рис.).

Многочисленные клинические исследования продемонстрировали высокую эффективность рабепразола для терапии ГЭРБ. Систематический анализ эффективности ИПП у пациентов с ГЭРБ показал максимальное влияние рабепразола на средние 24-часовые показатели $\mathrm{pH}$ в желудке в сравнении с пантопразолом, лансопразолом, омепразолом и эзомепразолом [13]. Клиническое исследование у 288 пациентов с неэрозивной рефлюксной болезнью установило, что рабепразол в дозе 10 мг/сут был более эффективен для купирования изжоги в сравнении с плацебо. Результаты лечения не зависили от наличия ожирения и полиморфизма в системе цитохрома [14]. Группа исследователей из США показала, что уже через 4 недели применения рабепразола у 87,5\% пациентов исчезает дневная изжога, у 90,7\% лиц - ночная изжога [15]. В 5-летнем проспективном плацебо-контролируемом исследовании в США у 497 человек частота рецидивов эзофагита была достоверно снижена в конце терапии рабепразолом в дозе 10 мг и 20 мг, в сравнении с группой плацебо и составляла на дозе 20 мг - 4\%, на дозе 10 мг - 23\% ( $p=0,005)$, на плацебо - 63\% ( $<$ 0,001). Рабепразол хорошо переносился. Частота неблагоприятных явлений после 5 лет применения существенно не отличались в группах рабепразола и плацебо [16]. Отечественные авторы согласны с точкой зрения, что рабепразол является средством выбора у пациентов с рефрактерной ГЭРБ, позволяя достигать хороших результатов у больных с ночными симптомами, у лиц с ожирением и полиморфизмом в системе цитохрома [17].

\section{РАБЕПРАЗОЛ И ПРОФИЛАКТИКА НПВП-ГАСТРОПАТИЙ}

Возникновение гастродуоденальных кровотечений является частым осложнением систематического применения НПВП или антитромбоцитарных средств для профилактики сердечно-сосудистых заболеваний. Важным принципом профилактики этих осложнений является назначение ИПП [18]. В контексте этого положения большое значение имеет метаболизм в организме различных ИПП. Современные работы показали, что рабепразол метаболизируется энзиматическим путем, практически не вступает в лекарственное взаимодействие, тогда как омепразол метаболизируется преимущественно в системе CYP2C19, в связи с чем его влияние на метаболизм клопидогрела является весьма значительным [19]. В клиническом исследовании у 218 пациентов рабепразол в дозе 20 мг/сут показал высокую эффективность для профилактики гастродуоденальных осложнений у пациентов, получавших аспирин или клопидогрел [20]. В этой связи назначение рабепразола является предпочтительным у пациен-

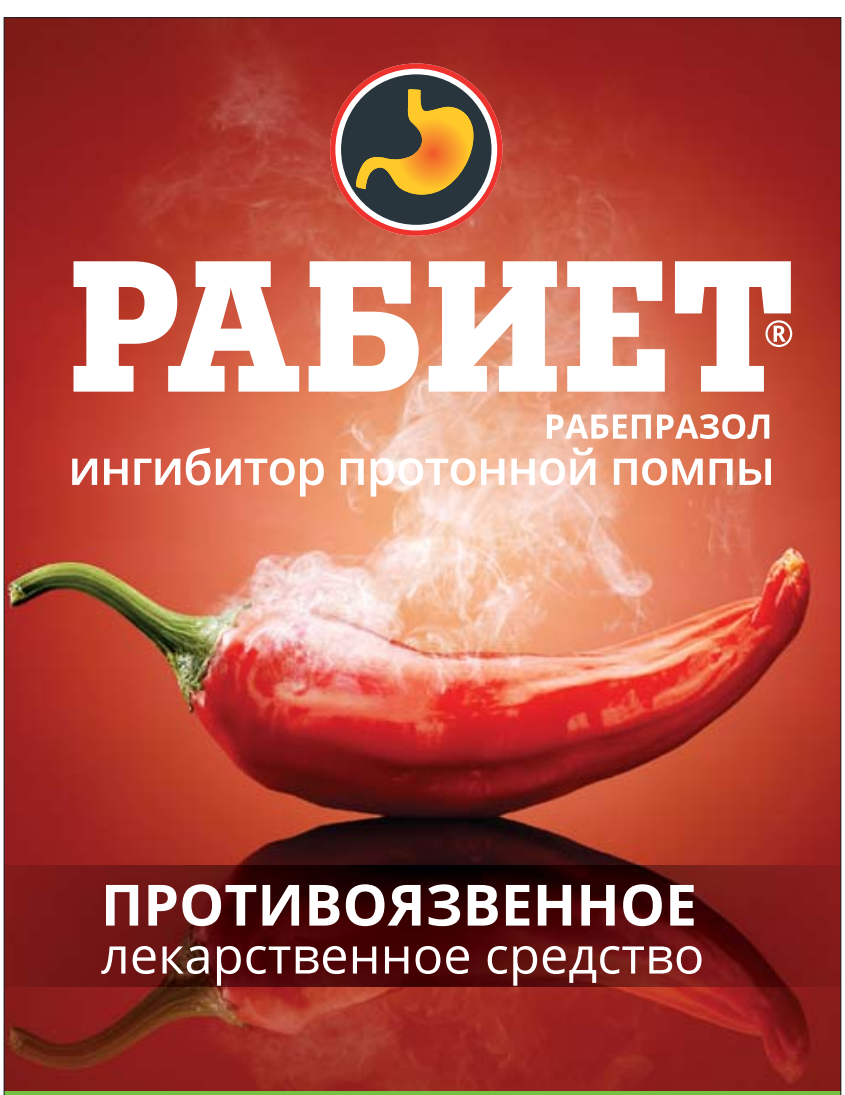

10 мг капс. №14, 20 мг капс. №14, 20 мг капс. №28

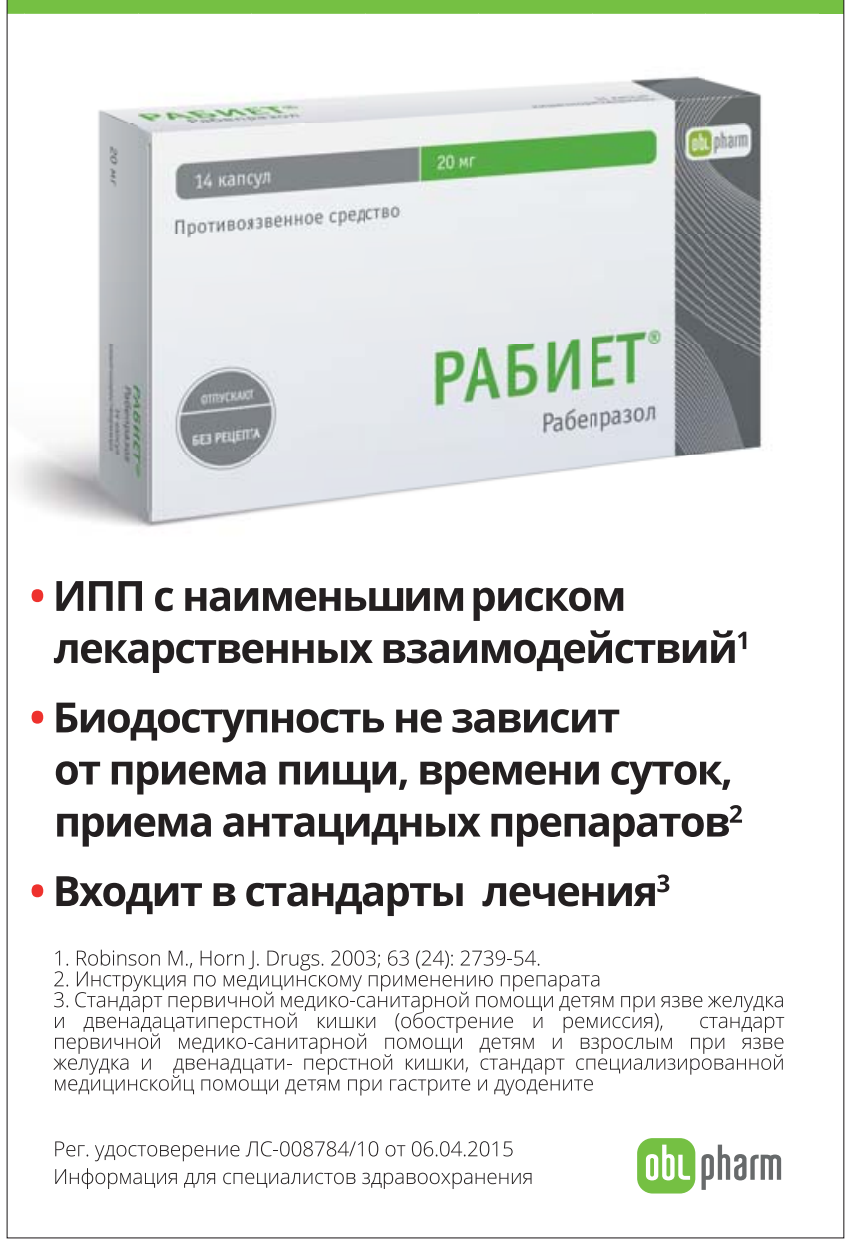


Тов, принимающих НПВП, аспирин или клопидогрел [21].

Одним из эффективных дженериков рабепразола является препарат Рабиет ${ }^{\circledast}$. Официальными показаниями для назначения препарата являются язвенная болезнь желудка в фазе обострения и язва анастомоза; язвенная болезнь двенадцатиперстной кишки в фазе обострения; эрозивная и язвенная гастроэзофагеальная рефлюксная болезнь или рефлюкс-эзофагит; поддерживающая терапия гастроэзофагеальной рефлюксной болезни; неэрозивная гастроэзофагеальная рефлюксная болезнь; синдром Золлингера - Эллисона и другие состояния, характеризующиеся патологической гиперсекрецией; антибактериальная терапия для эрадикации Helicobacter pylori.

\section{Рисунок. Алгоритм лечения пациентов с рефрактерной ГЭРБ [11]}

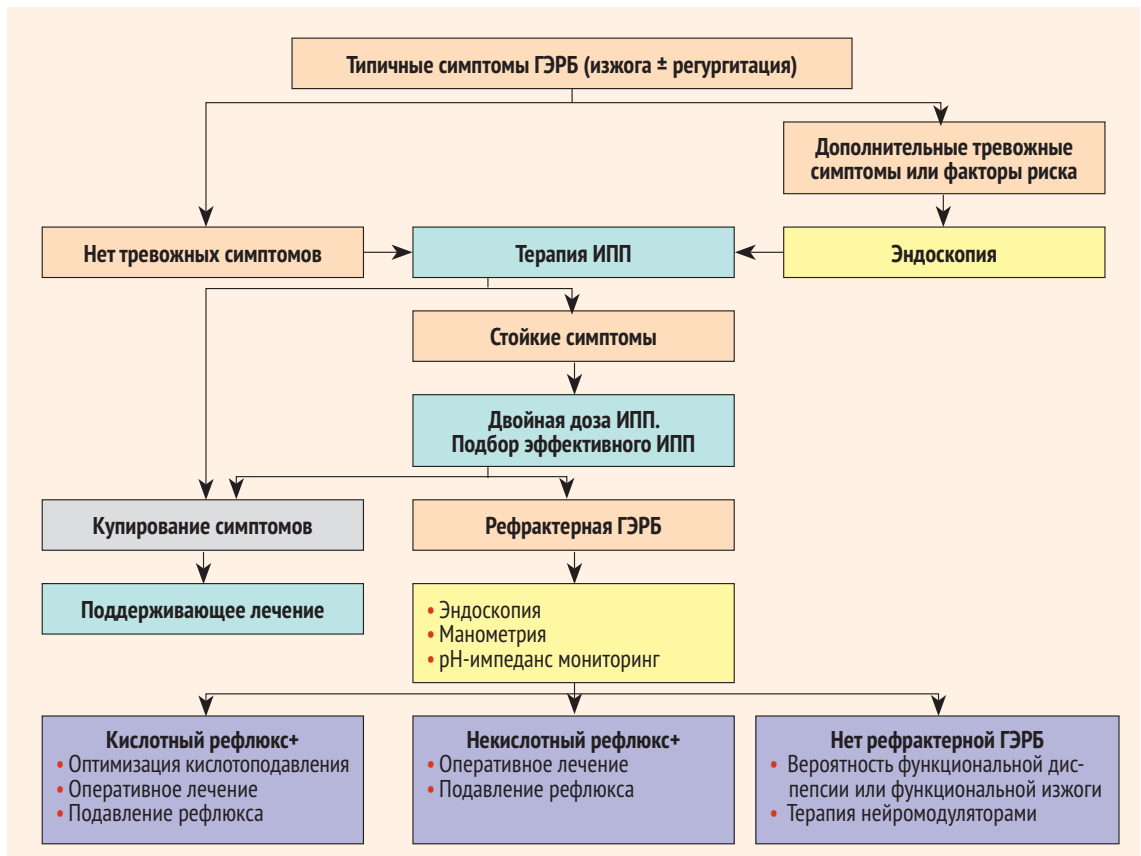

\section{ЗАКЛЮЧЕНИЕ}

Рабепразол является ИПП второго поколения, имеющим преимущественно энзиматический путь метаболизма, что позволяет ему достигать высокой эффективности у больных с полиморфизмом в системе СҮР2С19. Следствием этого являются хорошие результаты в эрадикации Helicobacter pylori [7]. Клинические исследования подтвердили высокую эффективность рабепразола для лечения ГЭРБ и профилактики НПВП-гастропатий [22]. На российском рынке современным и эффективным дженериком рабепразола является препарат Рабиет ${ }^{\circledR}$. Он производится по стандартам GMP и имеет доступную цену для потребителя.

\section{ЛИТЕРАТУРА}

1. Агеева Е.С., Штыгашева О.В., Рязанцева Н.В., Цуканов В.В. Молекулярно-генетические фак торы, влияющие на исход инфицирования Helicobacter pylori у жителей Республики Хакасия. Рос. журн. гастроэнтерологии, гепатологии, колопроктологии, 2010, 20(4): 16-21.

2. Meyer UA. Metabolic interactions of the protonpump inhibitors lansoprazole, omeprazole and pantoprazole with other drugs. Eur J Gastroenterol Hepatol, 1996, 8(Suppl 1): S21-S25.

3. Zhao F, Wang J, Yang Y, Wang X, Shi R, Xu Z et al. Effect of CYP2C19 genetic polymorphisms on the efficacy of proton pump inhibitor-based triple therapy for Helicobacter pylori eradication: a meta-analysis. Helicobacter, 2008, 13(6): 532-541.

4. Kuo CH, Lu CY, Shih HY, Liu CJ, Wu MC, Hu HM et al. CYP2C19 polymorphism influences Helicobacter pylori eradication. World J Gastroenterol, 2014, 20(43): 16029-16036.

5. McNicholl AG, Linares PM, Nyssen OP, Calvet $X$ Gisbert JP. Meta-analysis: esomeprazole or rabeprazole vs. first-generation pump inhibitors in the treatment of Helicobacter pylori infection. Aliment Pharmacol Ther, 2012, 36(5): 414-425.

6. Fujioka T, Aoyama N, Sakai K, Miwa Y, Kudo M, Kawashima J et al. A large-scale nationwide multicenter prospective observational study of triple therapy using rabeprazole, amoxicillin, and clarithromycin for Helicobacter pylori eradication in Japan. J Gastroenterol, 2012, 47(3): 276-283.

7. Malfertheiner P, Megraud F, O'Morain CA Gisbert JP, Kuipers EJ, Axon AT et al.
Management of Helicobacter pylori infectionthe Maastricht V/Florence Consensus Report. Gut, 2017, 66(1): 6-30.

8. Буторин Н.Н., Бичурина Т.Б., Цуканов В.В., Каспаров Э.В., Куклин Д.В., Тимошенко В.О. и др. Распространенность и клинические аспекты пищевода Барретта у населения Восточной Сибири. Терапевт. архив, 2013, 85(1): 62-65.

9. Цуканов В.В., Онучина Е.В., Васютин А.В., Буторин Н.Н., Амельчугова О.С. Клинические аспекты гастроэзофагальной рефлюксной болезни у лиц пожилого возраста: результаты 5-летнего проспективного исследования. Терапевт. архив, 2014, 86(2): 23-26.

10. Цуканов В.В., Каспаров Э.В., Онучина Е.В., Васютин А.В., Буторин Н.Н., Амельчугова О.С. Тонких Ю.Л. Частота и клинические аспекты внепищеводных синдромов у пациентов с гастроэзофагеальной рефлюксной болезнью пожилого возраста. Терапевт. архив, 2016, 88(2): 28-32.

11. Scarpellini E, Ang D, Pauwels A, De Santis A, Vanuytsel T, Tack J. Management of refractory typical GERD symptoms. Nat Rev Gastroenterol Hepatol, 2016, 13(5): 281-294.

12. Fass R, Gasiorowska A. Refractory GERD: what is it? Curr Gastroenterol Rep, 2008, 10(3): 252-257.

13. Kirchheiner J, Glatt S, Fuhr U, Klotz U, Meineke I, Seufferlein T, Brockmöller J. Relative potency of proton-pump inhibitors-comparison of effects on intragastric pH. Eur J Clin Pharmacol, 2009, 65(1): 19-31.

14. Kinoshita $\mathrm{Y}$, Ashida $\mathrm{K}$, Hongo $\mathrm{M}$ et al. Randomised clinical trial: a multicentre, double-blind, placebo-controlled study on the efficacy and safety of rabeprazole $5 \mathrm{mg}$ or $10 \mathrm{mg}$ once daily in patients with non-erosive reflux disease. Aliment Pharmacol Ther, 2011, 33(2): 213-224.

15. Cutler A, Robinson M, Murthy A, Delemos B. Rabeprazole $20 \mathrm{mg}$ for erosive esophagitis-associated symptoms in a large, community-based study: additional results. Dig Dis Sci, 2010, 55(2): 338-345.

16. Caos A, Breiter J, Perdomo C, Barth J. Long-term prevention of erosive or ulcerative gastrooesophageal reflux disease relapse with rabeprazole 10 or $20 \mathrm{mg}$ vs. placebo: results of a 5 -year study in the United States. Aliment Pharmacol Ther, 2005, 22(3): 193-202.

17. Евсютина Ю.В., Трухманов А.С. Недостаточный ответ на терапию ингибиторами протонного насоса: причины и тактика ведения пациентов. Терапевт. архив, 2015, 87(2): 85-89.

18. Каратеев А.Е., Насонов Е.Л., Яхно Н.Н., Ивашкин В.Т., Чичасова Н.В., Алексеева Л.И. и др. Клинические рекомендации «Рациональное применение нестероидных противовоспалительных препаратов (НПВП) в клинической практике». Современная ревматология, 2015, (1): 4-23.

19. Wedemeyer RS, Blume H. Pharmacokinetic drug interaction profiles of proton pump inhibitors: an update. Drug Saf, 2014, 37(4): 201-211.

20. Chan FK, Ching JY, Hung LC, Wong VW, Leung VK, Kung NN et al. Clopidogrel versus aspirin and esomeprazole to prevent recurrent ulcer bleeding. N Engl J Med, 2005, 352(3): 238-244.

21. Wang ZY, Chen M, Zhu LL, Yu LS, Zeng S, Xiang MX, Zhou Q. Pharmacokinetic drug interactions with clopidogrel: updated review and risk management in combination therapy. Ther Clin Risk Manag, 2015, 11: 449-467.

22. Marelli S, Pace F. Rabeprazole for the treatment of acid-related disorders. Expert Rev Gastroenterol Hepatol, 2012, 6(4): 423-435. 